\title{
Adventitial Activation in the Pathogenesis of Injury-Induced Arterial Remodeling
}

\section{Potential Implications in Transplant Vasculopathy}

\author{
Jianli Wang, ${ }^{*}$ Yuan Wang, ${ }^{\dagger}$ Jingjing Wang, ${ }^{*}$ Xiaosun Guo, ${ }^{*}$ Elsa C. Chan,${ }^{\ddagger}$ and Fan Jiang $* \S \varpi$
}

From the Department of Physiology and Pathophysiology, ${ }^{*}$ School of Basic Medicine, Shandong University, Jinan, China; the Department of Emergency, ${ }^{\dagger}$ the Key Laboratory of Cardiovascular Remodeling and Function Research (Chinese Ministry of Education and Chinese Ministry of Health), ${ }^{\S}$ and The State and Shandong Province Joint Key Laboratory of Translational Cardiovascular Medicine, Qilu Hospital of Shandong University, Jinan, China; and the Centre for Eye Research Australia, ${ }^{\ddagger}$ Royal Victorian Eye and Ear Hospital, East Melbourne, Victoria, Australia

\author{
Accepted for publication \\ December 7, 2017. \\ Address correspondence to \\ Fan Jiang, Ph.D., Department of \\ Physiology and Pathophysi- \\ ology, School of Basic Medi- \\ cine, Shandong University, \\ 44 Wen Hua Xi Rd, Jinan, \\ Shandong Province 250012, \\ China. E-mail: fjiang@sdu. \\ edu.cn.
}

\begin{abstract}
Transplant vasculopathy is one of the major causes of chronic rejection after solid organ transplantation. The pathogenic mechanisms of transplant vasculopathy are still poorly understood. Herein, we summarize current evidence suggesting that activation of the tunica adventitia may be involved in the pathogenesis of transplant vasculopathy. Adventitia is an early responder to various vascular injuries and plays an integral role in eliciting vascular inflammation and remodeling. Accumulation of macrophages in the adventitia promotes the development of vascular remodeling by releasing a variety of paracrine factors that have profound impacts on vascular mural cells. Targeting adventitial macrophages has been shown to be effective for repressing transplantation-induced arterial remodeling in animal models. Adventitia also fosters angiogenesis, and neovascularization of the adventitial layer may facilitate the transport of inflammatory cells through the arterial wall. Further investigations are warranted to clarify whether inhibiting adventitial oxidative stress and/or adventitial neovascularization are better strategies for preventing transplant vasculopathy. (Am J Pathol 2018, 188: 838-845; https://doi.org/10.1016/j.ajpath.2017.12.002)
\end{abstract}

Acute rejection after solid organ transplantation is mainly mediated by activation of the classic adaptive immune responses involving $\mathrm{CD}^{+}$and $\mathrm{CD}^{+} \mathrm{T}$ lymphocytes and antibodies, ${ }^{1}$ which can be effectively inhibited by available immunosuppressive drugs. ${ }^{2}$ In contrast, immunosuppressive drugs have limited effects on the development of chronic rejection, which remains to be a major hurdle for long-term allograft survival and tolerance. ${ }^{3}$ The pathogenesis of chronic rejection is complex, involving both immune and nonimmune mechanisms, ${ }^{3}$ among which transplant vasculopathy is a fundamental pathologic change underlying the delayed failure of transplanted organs. ${ }^{4}$ End-stage transplant vasculopathy is characterized by severe diffuse intimal hyperplasia and arterial occlusion, for which replacing the entire allograft is currently the only treatment option. ${ }^{4}$ As a result, further understanding of the pathogenesis of transplant vasculopathy may provide a better means to improve long-term survival of the allograft.
Adventitia is the outer layer of the blood vessel wall, primarily serving as a supportive structure that anchors the vessel to surrounding tissues. In addition to most fibroblasts, adventitia also contains nerve endings, adipocytes, leukocytes, and some progenitor cells. Another important function of adventitia is to nourish the vessel wall via vasa vasorum. ${ }^{5}$ Similar to the mechanisms of neointima formation after arterial angioplasty, proliferation, migration, and synthesis of extracellular matrix by medial smooth muscle cells have a central role in the pathogenesis of transplant

\footnotetext{
Supported by National Natural Science Foundation of China research grants 91539102 (F.J.) and 31471087 (F.J.), National 973 Basic Research Program 2010CB732605 (F.J.), Natural Science Foundation of Shandong Province ZR2016HM24 (J.W.), and Innovation Fund of Ji'nan City 201401251 (J.W.).

Disclosures: None declared.
} 
Normal vessel
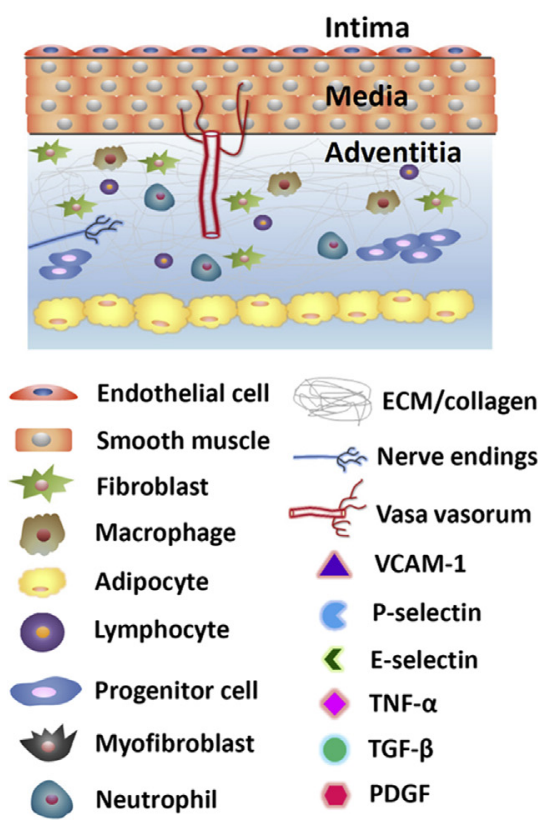

Adventitial activation
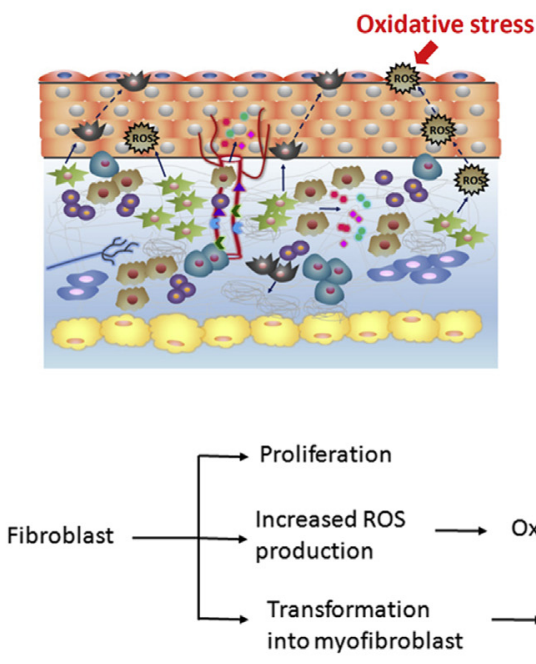

Progenitor cell migration

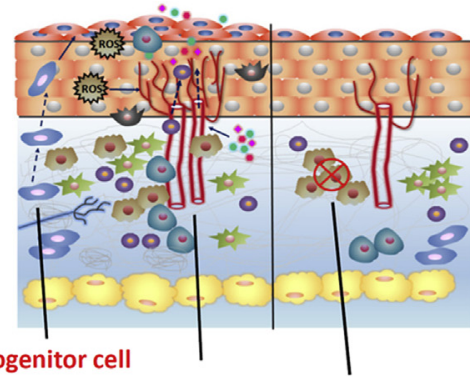

Adventitial Inhibition of angiogenesis macrophage accumulation

Increased

migration and

collagen synthesis

Infiltration of macrophages, Lymphocytes, and neutrophils

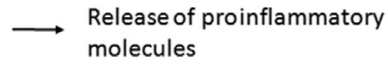

Figure 1 Activation of the tunica adventitia may have a crucial role in the pathogenesis of transplant vasculopathy. Adventitia is an early responder to various vascular injuries. Adventitia fosters neovascularization, and the newly formed vasa vasorum may facilitate the transport of inflammatory cells through the arterial wall. On inflammatory stimulation, adventitial progenitor cells start to migrate into the artery wall. Accumulation of macrophages results in the release of various proinflammatory paracrine factors from the adventitial layer. Other hallmarks of such an outside-in model include increased activation and migration of fibroblasts and myofibroblasts and enhanced adventitia-derived oxidative stress. It is suggested that targeting adventitial macrophage accumulation and activation may be an effective therapy for transplantation-induced arterial remodeling. ECM, extracellular matrix; PDGF, platelet-derived growth factor; ROS, reactive oxygen species; TGF- $\beta$, transforming growth factor- $\beta$; TNF- $\alpha$, tumor necrosis factor- $\alpha$; VCAM- 1 , vascular cell adhesion molecule- 1 .

vasculopathy. ${ }^{4}$ It has traditionally been known that the development of vascular lesions, such as atherosclerosis, follows an inside-out paradigm. This is mainly based on the observation that injuries to endothelial cells often occur before other morphologic changes in the vessel wall. Endothelial dysfunction then causes activation of medial smooth muscle cells, leading to neointima formation. In contrast, the adventitia has somehow been overlooked in this traditional concept. ${ }^{6,7}$

More than two decades ago, it was found that surgical removal of the adventitia in rabbit carotid artery induced intimal hyperplasia. ${ }^{8}$ This finding attracted other researchers to investigate the roles of adventitia in the development of vascular lesions. Since then, cumulative results have suggested that the adventitia is not merely a bystander in the pathogenesis of arterial diseases; rather, it may represent a direct driving force for the development of vascular lesions. ${ }^{6,7}$ Increasing evidence in recent years has also highlighted the importance of adventitial activation in the proliferation of vasa vasorum. ${ }^{9}$ Activated adventitia may be an unguarded gate that allows the intravasation of inflammatory cells into the media and intima via vaso vasorum. $^{10,11}$ At the same time, different types of cells located in the adventitia (including fibroblasts, macrophages, lymphocytes, stem cells, progenitor cells, and adipocytes) can release multiple factors, ${ }^{6,7,12}$ which regulate differentiation, proliferation, apoptosis, migration, and collagen synthesis of other cells through paracrine mechanisms. Therefore, the adventitia forms an ideal platform for cross talk among these cells, which is critical for the maintenance of vascular homeostasis, as well as the induction of vascular pathologies, such as inflammation, neointimal remodeling, and fibrosis.

Herein, we summarize current evidence highlighting a potential implication of adventitial activation in the pathogenesis of transplant vasculopathy. We hypothesize that an outside-in mechanism, as proposed by other researchers, may have a significant contribution in this process $^{7,9}$ (Figure 1). Potential mechanisms by which adventitial activation contributes to pathologic vascular remodeling have also been discussed.

\section{Adventitial Activation in the Early Phase of Transplant Vasculopathy}

Different injuries to blood vessels (including balloon angioplasty, stenting, luminal denudation, and hypercholesterolemia) trigger vascular remodeling, which features formation of a neointimal layer and loss of the vessel lumen. Several lines of evidence support that the adventitia encompasses the initial pathologic changes during injury-induced vascular remodeling. It was observed that in porcine coronary arteries with balloon injury, there was a 
rapid increase in cell proliferation in the adventitia, which reached the maximum level by 3 days; this proliferating activity in the adventitia exceeded that in the media at all time points after injury. ${ }^{13}$ Similarly, Scott et $\mathrm{al}^{14}$ found a large number of proliferating cells in the adventitia of porcine coronary arteries at 3 days after angioplasty. In contrast, there was little accumulation of proliferating cells in the media and intima until 7 days after injury. In a porcine model of hypercholesterolemia, researchers identified adventitial remodeling of the coronary artery (adventitial angiogenesis) as an early pathologic change that preceded the development of changes in intima and media. ${ }^{15}$ Kortelainen and Porvari ${ }^{16}$ analyzed human coronary artery specimens and found that immature atherosclerotic plaques were surrounded by numerous adventitial macrophages, indicating that the formation of atherosclerotic plaques was associated with early adventitial inflammation. The implication of initial adventitial activation in the pathogenesis of intimal hyperplasia is nicely shown by experiments using genetic labeling to trace the origin of cells. Using LacZ ( $\beta$-galactosidase) - transfected adventitial fibroblasts, $\mathrm{Li}$ et $\mathrm{al}^{17}$ identified LacZ-expressing fibroblasts in the neointima induced by arterial injury. In addition, arterial injury stimulates the transformation of adventitial fibroblasts into myofibroblasts, and some of these transdifferentiated cells can migrate into the intima through the medial layer. ${ }^{13,14}$ Apart from augmented proliferation and migration activities, phenotypically changed fibroblasts also have an increased capacity to secrete extracellular matrix. ${ }^{18}$ In a porcine model of renal hypertension, remodeling of coronary artery adventitia is accompanied by increases in both fibroblast proliferation and collagen III synthesis. ${ }^{15}$

In line with the previous observations, we and others have found that activation of the adventitial layer also occurs before the development of transplant vasculopathy. Mennander et al, ${ }^{19}$ in 1991, developed a useful experimental model for studying transplant vasculopathy by allografting aortic segments in rats. Using this model, these authors demonstrated that an acute inflammation phase in the adventitia occurred before the formation of neointima. ${ }^{19}$ This early inflammatory response was characterized by infiltration of both macrophages and lymphocytes into the adventitia, which could be seen as early as 12 hours after transplantation. ${ }^{20}$ The thickness of the adventitia and increases in fibroblast number reached the maximum level at 1 month after transplantation, whereas changes in the intimal cell number and thickness peaked at 12 months, ${ }^{19}$ suggesting that adventitial activation preceded pathologic changes of the intima during the course of transplant vasculopathy.

Using the same model, it was shown that $\alpha$-smooth muscle actin-positive cells started to accumulate in the adventitia 3 days after aortic transplantation, before the first appearance of neointima around 7 days. ${ }^{21}$ At 3 days, there were increases in cells that were positive for Ki-67 (a marker of cell proliferation), monocyte chemoattractant protein-1, and matrix metalloproteinase-7 in the adventitia; however, these cells appeared at a later time point in the intima. ${ }^{21}$ Consistently, the protein levels of monocyte chemoattractant protein-1 and other inflammatory cytokines, such as tumor necrosis factor- $\alpha$ and IL- $1 \beta$, were all increased in the adventitia at 3 days after transplantation. ${ }^{21}$ These data raise the possibility that the pathogenesis of transplant vasculopathy is an integrated process beginning from the adventitial layer. However, the aortic transplantation model of transplant vasculopathy might not be able to precisely reproduce the situation in human organ transplants, because the adventitial responsiveness to injury in large and small arteries may be different.

\section{Adventitia and Vascular Inflammation}

Under pathologic conditions, the adventitia is infiltrated with various kinds of inflammatory cells, including macrophages, lymphocytes, and neutrophils. These cells play important roles in the development of arterial remodeling. In 1915, Allbutt ${ }^{22}$ first described the accumulation of inflammatory cells in the adventitia adjacent to human atherosclerotic plaques. Schwartz and Mitchell ${ }^{23}$ analyzed 440 tissue samples from 111 cases of coronary artery disease and identified a positive correlation between the severity of atherosclerotic plaque and the number of adventitial inflammatory cells. Mounting evidence has suggested that in response to vascular injury, adventitia is often the first mural compartment to be activated, which then contributes to the perpetuation of chronic vascular inflammation. ${ }^{5,24}$ In particular, components of both innate and adaptive immunity systems have been identified in the activated inflaming adventitia. ${ }^{5,24}$ Using a porcine model of balloon-induced coronary artery injury, Okamoto et $\mathrm{al}^{11}$ discovered that neutrophil accumulation in the adventitia started at 2 hours after injury and lasted for 3 days. Macrophages also aggregated in the adventitia from day 1 to day 7. The induction of leukocyte accumulation appeared to be mediated by the expression of adhesion molecules, such as P-selectin, E-selectin, and vascular cell adhesion molecule-1, in the adventitia. ${ }^{11}$ Histologic examination of serial heart sections from apolipoprotein-E-deficient mice showed that before the appearance of spontaneous atherosclerotic lesions, the adventitia was infiltrated by profuse macrophages. ${ }^{25}$ For atherosclerotic disease, at least at early time points before intraplaque angiogenesis, the accumulation of leukocytes under the luminal endothelium is unlikely to be a result of massive transmural migration from the adventitia. Nonetheless, adventitial leukocytes may facilitate the recruitment of inflammatory cells into the subendothelial space by releasing various chemokines. ${ }^{\text {? }}$

During the development of transplant vasculopathy, adventitial inflammation is also apparent before the development of neointimal lesions in transplanted aortas. ${ }^{19,21}$ Adventitial inflammation may have a decisive role in the 
progression of luminal occlusion. Miller et $\mathrm{al}^{20}$ demonstrated that prophylaxis treatment with an anti-inflammatory serpin significantly reduced the size of intima after aortic transplantation. Adventitial inflammation is not only associated with activation of medial smooth muscle cells, it also affects residential fibroblasts in the adventitia. Arterial adventitial fibroblasts are sensitive to various pathologic stimuli. For example, hypoxia, hypercholesterolemia, and mechanical injury can all stimulate proliferation, migration, and transdifferentiation (into myofibroblasts) of adventitial fibroblasts. ${ }^{18}$ There are increases in the extent of proliferation and migration of fibroblasts isolated from the adventitia of transplanted aortas, as compared with those from sham-operated vessels. ${ }^{21,26}$ Although there is evidence indicating that migration of adventitial fibroblasts contributes to balloon injury-induced neointima formation, ${ }^{17}$ so far there is no clear evidence suggesting that the same mechanism applies to the development of transplant vasculopathy. Apart from immune cells, activated fibroblasts can also release proinflammatory cytokines and growth factors on stimulation, and such a release may even start before the recruitment of inflammatory cells. ${ }^{27,28}$

Although the nature of initial damage to vascular cells varies with different types of injuries, it has been established that vascular inflammation may act as a driving force in the development of subsequent neointimal remodeling. A fundamental role of vascular inflammation in transplant vasculopathy has been well recognized. ${ }^{29,30}$ Of note, a pivotal mechanism by which adventitia promotes vascular remodeling is to orchestrate the development of vascular inflammation. ${ }^{7,9}$ Therefore, inflammation may represent a central mechanistic link between adventitia activation and transplant vasculopathy.

\section{Critical Roles of Adventitial Macrophage in Transplant Vasculopathy}

A major cellular component of transplantation-induced adventitial inflammation is monocyte/macrophage. ${ }^{19,21} \mathrm{Li}$ et $\mathrm{al}^{27}$ demonstrated that vascular endothelial growth factor was expressed in the adventitia at an early stage of balloon injury-induced arterial remodeling before the expressions of vascular endothelial growth factor in the media and intima. With an intervention targeting on vascular endothelial growth factor expression, the accumulation of macrophages in the adventitial layer and the subsequent luminal occlusion were both decreased, highlighting a correlation between early adventitial macrophage infiltration and neointimal remodeling. ${ }^{27}$ Similarly, we and others have shown that systemic depletion of endogenous macrophages suppressed the development of transplant vasculopathy. ${ }^{31,32}$ It has been recognized that the initial phases of adventitial inflammatory cell infiltration are similar in both acute arterial injuries and chronic vascular diseases, like atherosclerosis. ${ }^{9}$ Mechanistically, monocytes/macrophages can secrete various cytokines and proinflammatory factors, including ILs, tumor necrosis factor- $\alpha$, transforming growth factor- $\beta$, and platelet-derived growth factor. These factors may stimulate the proliferation and migration of medial smooth muscle cells and adventitial fibroblasts. Macrophages can also secrete matrix metalloproteinases, which have critical roles in both vessel repair and remodeling by regulating the breakdown of extracellular matrix and facilitating migration of smooth muscle cells and fibroblasts.

In addition to smooth muscle cells and fibroblasts, interactions between macrophages and vascular progenitor cells have also been implicated in pathologic arterial remodeling. Recent studies have suggested that adventitia serves as a depot of heterogeneous progenitor cells, while it also provides a platform for interactions between the resident progenitors and trafficking leukocytes. ${ }^{6}$ Using the rat aortic allograft model, Grudzinska et $\mathrm{al}^{33}$ have provided evidence showing that one third of cells in the neointima are of an adventitial origin, and many of these cells express markers of mesenchymal stem cells, suggesting that adventitial progenitor cells can migrate to the intima. Similarly, in a murine aortic transplantation model, Sun et $\mathrm{al}^{34}$ also identified that directional migration of progenitor cells from the adventitia contributes to the pathogenesis of transplant vasculopathy. In vitro, progenitor cells isolated from the adventitia are able to differentiate into smooth muscle-like cells under stimulation of factors that are abundantly expressed by macrophages, such as plateletderived growth factor. ${ }^{33}$ Interestingly, Psaltis et $\mathrm{al}^{35}$ have reported that vascular adventitia contains a population of macrophage progenitor cells, which proliferate in response to hypercholesterolemia and have a durable contribution to macrophage progeny in the adventitia.

Increasing evidence has suggested that changes in the expression of certain miRNA molecules may have critical roles in neointimal remodeling after arterial injuries. ${ }^{36-38}$ Vascular miRNAs may exert their effects by modulating the proliferation, migration, and/or phenotype differentiation of vascular smooth muscle cells. Of the miRNAs related to vascular remodeling, miR-21 has been shown to be highly expressed in arterial smooth muscle cells, and the abundant expression of miR-21 has been implicated in vascular lesion formation. ${ }^{39}$ For instance, local infusion of an antisense construct to miR-21 reduced neointima formation in balloon-injured arteries. ${ }^{36}$ The expression of miR-21 in adventitia and media is increased over time after aorta transplantation. ${ }^{32}$ Adventitial fibroblasts isolated from the transplanted aorta were cultured, and an increased expression of miR-21 was observed in comparison to cells isolated from sham vessels. Moreover, treating transplanted vessels with miR-21 antagomir induced a significant reduction in the development of neointima and significantly reduced the inflammatory responses in both media and adventitia. ${ }^{32}$ Using in vitro assays, macrophages were found to stimulate miR-21 expression in smooth muscle cells and adventitial fibroblasts via the release of tumor necrosis 
factor- $\alpha$, whereas silencing of miR-21 expression attenuated tumor necrosis factor-induced proliferation, migration, and inflammation in smooth muscle cells and fibroblasts. ${ }^{32}$ More important, it was confirmed that systemic depletion of macrophages concomitantly repressed the vascular miR-21 expression and ameliorated transplantation-induced neointimal remodeling. These observations suggest that adventitial macrophages may promote transplant vasculopathy by regulating miRNA expressions in vascular mural cells via paracrine factors, and this process may represent a novel mechanism underlying the pathogenesis of transplant vasculopathy.

\section{Adventitial Activation and Vascular 0xidative Stress}

Under physiological conditions, the production and removal of reactive oxygen species (ROS) in vascular tissues remain in balance. However, such a balance is often compromised under pathologic conditions, such as atherosclerosis; as a result, oxidative stress occurs. NADPH oxidase is known to be one of the enzymes that mediates ROS production in the arterial wall. ${ }^{40}$ NADPH oxidase is a multimeric enzyme complex, and seven isoforms of the catalytic subunit have been identified in mammalian cells, known as Nox1, Nox2, Nox3, Nox4, Nox5, Duox1, and Duox2. Adventitial fibroblasts mainly express two isoforms (namely, Nox2 and Nox4), which have been shown to be the main sources of ROS in the vessel wall, causing vascular dysfunctions. ${ }^{40}$ Our study in the rat aortic allograft model has revealed that an augmented number of cells expressing NADPH oxidase is seen at the outer edge of the adventitial layer 3 days after transplantation. ${ }^{26}$ This change is associated with up-regulation in the expression of both Nox 2 and p47phox (an accessory subunit of NADPH oxidase). ${ }^{26}$ The early increase in Nox 2 expression in the adventitia may possibly be derived from infiltrating macrophages and resident adventitial fibroblasts because both cell types are known to express this isoform. Also, up-regulation of NADPH oxidase expression in the media and neointima occurred in a delayed manner, suggesting that, at least in this specific model of transplant vasculopathy, adventitial oxidative stress leads the change in other layers. ${ }^{26}$

Cumulative evidence suggests that NADPH oxidase-mediated adventitial oxidative stress may have crucial roles in the pathogenesis of vascular inflammation and remodeling. ${ }^{7}$ Adventitia-derived ROS (eg, hydrogen peroxide, in particular) can penetrate the vessel tissue and act as a paracrine factor, which regulates functions of endothelial and smooth muscle cells. ${ }^{41,42}$ This reductionoxidation mechanism may ultimately affect the vasomotor activities of blood vessels and participate in pathologic processes of neointimal hyperplasia and medial smooth muscle hypertrophy. ${ }^{41,42}$ A large body of experimental evidence shows that intervening adventitial oxidative stress can inhibit arterial remodeling and luminal occlusion. Dourron et $\mathrm{al}^{43}$ transfected balloon-injured arteries in rats with a virus expressing the Nox2 inhibitor peptide gp91ds-tat and found this treatment effectively prevented intimal thickening. Likewise, Chan et $\mathrm{al}^{44}$ administered gp91ds-tat to the adventitial layer of rabbit arteries implanted with a perivascular collar and demonstrated that Nox inhibition reduced ROS production and alleviated neointimal remodeling. Therefore, further studies are warranted to investigate whether targeting adventitial NADPH oxidase and oxidative stress is an effective therapy for transplant vasculopathy.

\section{Adventitial Angiogenesis}

Angiogenesis is important for wound healing responses in the body, but aberrant growth of new blood vessels is deleterious in pathologic conditions, such as diabetic retinopathy and tumorigenesis. Vascular injury is associated with adventitial neovascularization, and most of the current evidence indicates that an excessive angiogenic response in the adventitia is associated with more advanced vascular inflammation and remodeling. ${ }^{45}$ In terms of transplant vasculopathy, in a recent analysis performed in heart transplantation recipients, optical coherence tomography images taken at the left descending coronary artery 1 year after surgery revealed a significant correlation between the volume of adventitial vasa vasorum and the degree of neointimal remodeling. ${ }^{46}$ Indeed, it has been recognized that in inflamed vascular wall, hypoxia, inflammation, and neovascularization are intertwined pathologic processes and neovascularization may potentially contribute to vascular inflammation by facilitating leukocyte trafficking across the vessel wall. ${ }^{45}$

Several groups have characterized the relationship between adventitial angiogenesis and the development of neointimal remodeling in animal models of arterial injury. Pels et $\mathrm{al}^{47}$ found that in porcine coronary arteries subject to endothelium-denudation injury, the number and density of adventitial microvessels and the index of endothelial cell proliferation in the adventitia peaked at 3 days after injury, and this angiogenic response regressed gradually afterwards. In a similar study, Kwon et $\mathrm{al}^{48}$ observed the density of newly formed microvessels in the adventitia was proportional to vessel stenosis after balloon injury. Xu et $\mathrm{al}^{49}$ reported increases in the number of microvessels in the adventitia and expressions of proangiogenic factors (vascular endothelial growth factor and platelet-derived growth factor) in rat aortas with balloon injury. It was also found that there was a significant correlation between the level of adventitial angiogenesis and the degree of neointimal hyperplasia. Nevertheless, these data cannot prove a causal relationship between adventitial angiogenesis and neointimal remodeling; a possibility that neointimal hyperplasia may promote adventitial angiogenesis cannot be ruled out. 
Aiming to investigate the relationship between adventitial angiogenesis and transplantation-induced vascular remodeling, our laboratory recently evaluated the angiogenic response in rat aortic allografts using von Willebrand factor as a marker. Time-dependent increases were found in the number of microvessels in the adventitia at 7 and 14 days after transplantation. ${ }^{26} \mathrm{~A}$ significant correlation was observed between the number of adventitial microvessels and the average thickness of the neointima at day $14 .{ }^{26}$ The increased angiogenesis in adventitia may relate to the inflammatory response and oxidative stress induced by transplantation. There are concomitant elevations in the expressions of transforming growth factor- $\beta$ and NADPH oxidase in the adventitia early after transplantation. ${ }^{21,26}$ Transforming growth factor- $\beta$ is one of the major stimulators of NADPH oxidase expression in vascular cells. ${ }^{50}$ Transforming growth factor- $\beta$ can stimulate endothelial NADPH oxidase expression, which mediates ROS production and facilitates reduction-oxidation signaling to regulate proliferation and migration of endothelial cells and angiogenic responses in vitro. ${ }^{51,52}$ Moreover, NADPH oxidase has been shown to be a critical mediator of angiogenic responses in vivo. ${ }^{50}$ Interestingly, Thaunat et $\mathrm{al}^{53}$ showed that antiangiogenic therapy with ABT-510 (a nonapeptide thrombospondin analog), which had no direct immunomodulatory properties, significantly reduced adventitial angiogenesis in rat aortic transplants. This effect of ABT-510 was associated with a reduction of inflammatory cell infiltration and adventitial remodeling. Taken together, these results suggest that adventitial angiogenesis might represent a novel target for the treatment of transplant vasculopathy.

\section{Conclusions}

The conventional paradigm, which emphasizes that vascular remodeling is initiated by endothelial injuries triggering signal propagation in an outward way through the arterial wall, may be an oversimplified model that overlooks the importance of adventitia in eliciting vascular inflammation. ${ }^{7}$ Mounting evidence suggests that the tunica adventitia is an early responder to various vascular injuries and may play an integral role in eliciting vascular inflammation and remodeling. Adventitia fosters angiogenesis, and neovascularization of the adventitial layer may facilitate the transport of inflammatory cells through the arterial wall. On inflammatory stimulation, adventitial progenitor cells start to migrate into the artery wall, and this process is apt to contribute to the subsequent neointimal remodeling. Most important, accumulation of macrophages in the adventitia may promote the progress of transplant vasculopathy by releasing a variety of paracrine factors with profound impacts on vascular mural cells. Hallmarks of such an outside-in model include increased activation and migration of fibroblasts and myofibroblasts, increased adventitial neovascularization, increased adventitial infiltration of leukocytes, and enhanced adventitia-derived ROS generation. ${ }^{7}$ Interestingly, our studies with aorta transplantation have revealed that all of these features are present in the adventitia of the graft, and many of these events occur before the beginning of neointimal hyperplasia. ${ }^{21,26}$ Moreover, our results have demonstrated that targeting adventitial macrophage accumulation and activation may effectively repress transplantation-induced arterial remodeling. ${ }^{32}$ It is suggested that activation of the tunica adventitia may have crucial implications in the pathogenesis of transplant vasculopathy (Figure 1). Further investigations are warranted to clarify whether inhibiting adventitial NADPH oxidase and the process of neovascularization may represent new strategies for preventing this disease.

\section{References}

1. Alegre ML, Florquin S, Goldman M: Cellular mechanisms underlying acute graft rejection: time for reassessment. Curr Opin Immunol 2007, 19:563-568

2. Lindenfeld J, Miller GG, Shakar SF, Zolty R, Lowes BD, Wolfel EE, Mestroni L, Page RL 2nd, Kobashigawa J: Drug therapy in the heart transplant recipient, part II: immunosuppressive drugs. Circulation 2004, 110:3858-3865

3. Wedel J, Bruneau S, Kochupurakkal N, Boneschansker L, Briscoe DM: Chronic allograft rejection: a fresh look. Curr Opin Organ Transplant 2015, 20:13-20

4. Mitchell RN, Libby P: Vascular remodeling in transplant vasculopathy. Circ Res 2007, 100:967-978

5. Stenmark KR, Yeager ME, El Kasmi KC, Nozik-Grayck E, Gerasimovskaya EV, Li M, Riddle SR, Frid MG: The adventitia: essential regulator of vascular wall structure and function. Annu Rev Physiol 2013, 75:23-47

6. Majesky MW, Dong XR, Hoglund V, Mahoney WM Jr, Daum G: The adventitia: a dynamic interface containing resident progenitor cells. Arterioscler Thromb Vasc Biol 2011, 31:1530-1539

7. Meijles DN, Pagano PJ: Nox and inflammation in the vascular adventitia. Hypertension 2016, 67:14-19

8. Barker SG, Tilling LC, Miller GC, Beesley JE, Fleetwood G, Stavri GT, Baskerville PA, Martin JF: The adventitia and atherogenesis: removal initiates intimal proliferation in the rabbit which regresses on generation of a "neoadventitia". Atherosclerosis 1994, 105:131-144

9. Maiellaro K, Taylor WR: The role of the adventitia in vascular inflammation. Cardiovasc Res 2007, 75:640-648

10. Akhavanpoor $\mathrm{M}$, Wangler $\mathrm{S}$, Gleissner CA, Korosoglou G, Katus HA, Erbel C: Adventitial inflammation and its interaction with intimal atherosclerotic lesions. Front Physiol 2014, 5:296

11. Okamoto E, Couse T, De Leon H, Vinten-Johansen J, Goodman RB, Scott NA, Wilcox JN: Perivascular inflammation after balloon angioplasty of porcine coronary arteries. Circulation 2001, 104: $2228-2235$

12. Henrichot E, Juge-Aubry CE, Pernin A, Pache JC, Velebit V, Dayer JM, Meda P, Chizzolini C, Meier CA: Production of chemokines by perivascular adipose tissue: a role in the pathogenesis of atherosclerosis? Arterioscler Thromb Vasc Biol 2005, 25:2594-2599

13. Shi Y, Pieniek M, Fard A, O'Brien J, Mannion JD, Zalewski A: Adventitial remodeling after coronary arterial injury. Circulation 1996, 93:340-348

14. Scott NA, Cipolla GD, Ross CE, Dunn B, Martin FH, Simonet L, Wilcox JN: Identification of a potential role for the adventitia in vascular lesion formation after balloon overstretch injury of porcine coronary arteries. Circulation 1996, 93:2178-2187 
15. Herrmann J, Samee S, Chade A, Rodriguez Porcel M, Lerman LO, Lerman A: Differential effect of experimental hypertension and hypercholesterolemia on adventitial remodeling. Arterioscler Thromb Vasc Biol 2005, 25:447-453

16. Kortelainen ML, Porvari K: Adventitial macrophage and lymphocyte accumulation accompanying early stages of human coronary atherogenesis. Cardiovasc Pathol 2014, 23:193-197

17. Li G, Chen SJ, Oparil S, Chen YF, Thompson JA: Direct in vivo evidence demonstrating neointimal migration of adventitial fibroblasts after balloon injury of rat carotid arteries. Circulation 2000, 101:1362-1365

18. Patel S, Shi Y, Niculescu R, Chung EH, Martin JL, Zalewski A: Characteristics of coronary smooth muscle cells and adventitial fibroblasts. Circulation 2000, 101:524-532

19. Mennander A, Tiisala S, Halttunen J, Yilmaz S, Paavonen T, Hayry P: Chronic rejection in rat aortic allografts: an experimental model for transplant arteriosclerosis. Arterioscler Thromb 1991, 11: $671-680$

20. Miller LW, Dai E, Nash P, Liu L, Icton C, Klironomos D, Fan L, Nation PN, Zhong R, McFadden G, Lucas A: Inhibition of transplant vasculopathy in a rat aortic allograft model after infusion of anti-inflammatory viral serpin. Circulation 2000, 101: $1598-1605$

21. Ji J, Xu F, Li L, Chen R, Wang J, Hu WC: Activation of adventitial fibroblasts in the early stage of the aortic transplant vasculopathy in rat. Transplantation 2010, 89:945-953

22. Allbutt TC: Diseases of the Arteries Including Angina Pectoris, vol II London: MacMillan and Co., Ltd., 1915. pp. 187

23. Schwartz CJ, Mitchell JR: Cellular infiltration of the human arterial adventitia associated with atheromatous plaques. Circulation 1962, 26:73-78

24. Michel JB, Thaunat O, Houard X, Meilhac O, Caligiuri G, Nicoletti A: Topological determinants and consequences of adventitial responses to arterial wall injury. Arterioscler Thromb Vasc Biol 2007, 27:1259-1268

25. Wang JL, Ma SQ, Li L, Liu GQ, Hu WC, Ma R: Correlation of inflammatory cells in adventitia and formation and extending of atherosclerotic lesions in coronary artery of apolipoprotein $\mathrm{E}$ gene knockout mice. Chin J Physiol 2013, 56:77-82

26. Sun M, Ji J, Guo X, Liu W, Wang Y, Ma S, Hu W, Wang J, Jiang F: Early adventitial activation characterized by NADPH oxidase expression and neovascularization in an aortic transplantation model. Exp Mol Pathol 2016, 100:67-73

27. Li XD, Chen J, Ruan CC, Zhu DL, Gao PJ: Vascular endothelial growth factor-induced osteopontin expression mediates vascular inflammation and neointima formation via Flt-1 in adventitial fibroblasts. Arterioscler Thromb Vasc Biol 2012, 32:2250-2258

28. Enzerink A, Vaheri A: Fibroblast activation in vascular inflammation. J Thromb Haemost 2011, 9:619-626

29. Shimizu K, Mitchell RN: The role of chemokines in transplant graft arterial disease. Arterioscler Thromb Vasc Biol 2008, 28: 1937-1949

30. Mitchell RN: Graft vascular disease: immune response meets the vessel wall. Annu Rev Pathol 2009, 4:19-47

31. Kitchens WH, Chase CM, Uehara S, Cornell LD, Colvin RB, Russell PS, Madsen JC: Macrophage depletion suppresses cardiac allograft vasculopathy in mice. Am J Transplant 2007, 7: 2675-2682

32. Guo X, Sun M, Dai C, Zhang X, Yin Q, Ling J, Li X, Wu X, Jiang F, Wang J: Macrophage-stimulated microRNA expression in mural cells promotes transplantation-induced neointima formation. Oncotarget 2017, 8:30100-30111

33. Grudzinska MK, Kurzejamska E, Bojakowski K, Soin J, Lehmann MH, Reinecke H, Murry CE, Soderberg-Naucler C, Religa P: Monocyte chemoattractant protein 1-mediated migration of mesenchymal stem cells is a source of intimal hyperplasia. Arterioscler Thromb Vasc Biol 2013, 33:1271-1279
34. Sun Y, Wang K, Ye P, Wu J, Ren L, Zhang A, Huang X, Deng P, Wu C, Yue Z, Chen Z, Ding X, Chen J, Xia J: MicroRNA-155 promotes the directional migration of resident smooth muscle progenitor cells by regulating monocyte chemoattractant protein 1 in transplant arteriosclerosis. Arterioscler Thromb Vasc Biol 2016, 36 : $1230-1239$

35. Psaltis PJ, Puranik AS, Spoon DB, Chue CD, Hoffman SJ, Witt TA, Delacroix S, Kleppe LS, Mueske CS, Pan S, Gulati R, Simari RD: Characterization of a resident population of adventitial macrophage progenitor cells in postnatal vasculature. Circ Res 2014, 115: 364-375

36. Ji R, Cheng Y, Yue J, Yang J, Liu X, Chen H, Dean DB, Zhang C: MicroRNA expression signature and antisense-mediated depletion reveal an essential role of MicroRNA in vascular neointimal lesion formation. Circ Res 2007, 100:1579-1588

37. McDonald RA, White KM, Wu J, Cooley BC, Robertson KE, Halliday CA, McClure JD, Francis S, Lu R, Kennedy S, George SJ, Wan S, van Rooij E, Baker AH: miRNA-21 is dysregulated in response to vein grafting in multiple models and genetic ablation in mice attenuates neointima formation. Eur Heart J 2013, 34 $1636-1643$

38. Yang Z, Zheng B, Zhang Y, He M, Zhang XH, Ma D, Zhang RN, Wu XL, Wen JK: miR-155-dependent regulation of mammalian sterile 20-like kinase 2 (MST2) coordinates inflammation, oxidative stress and proliferation in vascular smooth muscle cells. Biochim Biophys Acta 2015, 1852:1477-1489

39. Wei Y, Schober A, Weber C: Pathogenic arterial remodeling: the good and bad of microRNAs. Am J Physiol Heart Circ Physiol 2013 304:H1050-H1059

40. Drummond GR, Selemidis S, Griendling KK, Sobey CG: Combating oxidative stress in vascular disease: NADPH oxidases as therapeutic targets. Nat Rev Drug Discov 2011, 10:453-471

41. Ardanaz N, Pagano PJ: Hydrogen peroxide as a paracrine vascular mediator: regulation and signaling leading to dysfunction. Exp Biol Med (Maywood) 2006, 231:237-251

42. Haurani MJ, Pagano PJ: Adventitial fibroblast reactive oxygen species as autacrine and paracrine mediators of remodeling: bellwether for vascular disease? Cardiovasc Res 2007, 75: 679-689

43. Dourron HM, Jacobson GM, Park JL, Liu J, Reddy DJ, Scheel ML, Pagano PJ: Perivascular gene transfer of NADPH oxidase inhibitor suppresses angioplasty-induced neointimal proliferation of rat carotid artery. Am J Physiol Heart Circ Physiol 2005, 288: H946-H953

44. Chan EC, Datla SR, Dilley R, Hickey H, Drummond GR, Dusting GJ: Adventitial application of the NADPH oxidase inhibitor apocynin in vivo reduces neointima formation and endothelial dysfunction in rabbits. Cardiovasc Res 2007, 75:710-718

45. van Hinsbergh VW, Eringa EC, Daemen MJ: Neovascularization of the atherosclerotic plaque: interplay between atherosclerotic lesion, adventitia-derived microvessels and perivascular fat. Curr Opin Lipidol 2015, 26:405-411

46. Aoki T, Rodriguez-Porcel M, Matsuo Y, Cassar A, Kwon TG, Franchi F, Gulati R, Kushwaha SS, Lennon RJ, Lerman LO, Ritman EL, Lerman A: Evaluation of coronary adventitial vasa vasorum using $3 \mathrm{D}$ optical coherence tomography-animal and human studies. Atherosclerosis 2015, 239:203-208

47. Pels K, Labinaz M, Hoffert C, O'Brien ER: Adventitial angiogenesis early after coronary angioplasty: correlation with arterial remodeling. Arterioscler Thromb Vasc Biol 1999, 19:229-238

48. Kwon HM, Sangiorgi G, Ritman EL, Lerman A, McKenna C, Virmani R, Edwards WD, Holmes DR, Schwartz RS: Adventitial vasa vasorum in balloon-injured coronary arteries: visualization and quantitation by a microscopic three-dimensional computed tomography technique. J Am Coll Cardiol 1998, 32:2072-2079

49. Xu X, Lu H, Lin H, Li X, Ni M, Sun H, Li C, Jiang H, Li F, Zhang M, Zhao Y, Zhang Y: Aortic adventitial angiogenesis and 
lymphangiogenesis promote intimal inflammation and hyperplasia. Cardiovasc Pathol 2009, 18:269-278

50. Peshavariya HM, Chan EC, Liu GS, Jiang F, Dusting GJ: Transforming growth factor-beta1 requires NADPH oxidase 4 for angiogenesis in vitro and in vivo. J Cell Mol Med 2014, 18:1172-1183

51. Datla SR, Peshavariya H, Dusting GJ, Mahadev K, Goldstein BJ, Jiang F: Important role of Nox4 type NADPH oxidase in angiogenic responses in human microvascular endothelial cells in vitro. Arterioscler Thromb Vasc Biol 2007, 27:2319-2324
52. Peshavariya H, Dusting GJ, Jiang F, Halmos LR, Sobey CG, Drummond GR, Selemidis S: NADPH oxidase isoform selective regulation of endothelial cell proliferation and survival. Naunyn Schmiedebergs Arch Pharmacol 2009, 380:193-204

53. Thaunat O, Louedec L, Graff-Dubois S, Dai J, Groyer E, YacoubYoussef H, Mandet C, Bruneval P, Kaveri S, Caligiuri G, Germain S, Michel JB, Nicoletti A: Antiangiogenic treatment prevents adventitial constrictive remodeling in graft arteriosclerosis. Transplantation 2008, 85:281-289 